\title{
Heuristic and Eulerian Interface Capturing Approaches for Shallow Water Type Flow and Application to Granular Flows
}

\author{
Hossein Aghakhani ${ }^{\mathrm{a}}$, Keith Dalbey ${ }^{\mathrm{b}}$, David Salac ${ }^{\mathrm{a}}$, Abani K. Patra ${ }^{\mathrm{a}, *}$ \\ ${ }^{a}$ Department of Mechanical and Aerospace Engineering, University at Buffalo, Buffalo, New \\ York, United States \\ ${ }^{b}$ Sandia National Laboratories, Albuquerque, New Mexico, United States
}

\begin{abstract}
Determining the wet-dry boundary and avoiding the related spurious thin-layer problem when solving the depth-averaged shallow-water (SW) equations (or similar granular flow models) remains an outstanding challenge, though it has been the focus of much research effort. In this paper, we introduce the use of level set and phase field based methods to address this issue and related problems. We also propose new heuristic methods to address this problem. We implemented all of these methods in TITAN2D, which is a parallel adaptive mesh refinement toolkit designed for numerical simulation of granular flows. Results of the methods for flow over a simple inclined plane and Colima volcano are used to illustrate the methods. For the inclined plane, we compared the results with experimental data and for Colima volcano they are compared to field data. Our approaches successfully captured the interface of the flow and solved the accuracy and stability problems related to the thin layer problem in SW numerical solution. The comparison of results shows that although all of the methods can be used to address this problem, each of them has its own advantages/disadvantages and methods have to be chosen carefully for each problem.
\end{abstract}

Keywords:

\footnotetext{
${ }^{*}$ Corresponding author

Email address: abani@buffalo.edu (Abani K. Patra)
}

Preprint submitted to CMAME

January 23, 2016

(C) 2016. This manuscript version is made available under the Elsevier user license http://www.elsevier.com/open-access/userlicense/1.0/ 
Shallow water flow, Thin layer, Wetting/Drying, Phase field, Level Set

\section{Introduction}

Shallow water (SW) flows include a wide range of fluid flows in which the fluid depth is much smaller $\left(\mathcal{O}\left(10^{-1}\right)\right)$ than the characteristic length of the fluid body. The shallowness of flow allows us to approximate the variation of state 5 variables in the direction perpendicular to the basal surface by an integrated average [1, which thus reduces a three dimension flow problem into a twodimensional one. This approximation holds for many geophysical flows and the same conservation equations with minor variations can be used to study different physical situations. Eglit and Sveshnikova [2] modified the depth-averaged Saint

10 Venant equations for water flows to simulate granular snow avalanches and almost a decade later Savage and Hutter [1] popularized these in the modeling of many geophysical mass flows related to landslides, avalanches and debris flows. Since this type of flow has free moving boundaries, identifying the location of the flow interface is a critical challenge for successful numerical methods. Furthermore, the governing equations are valid only in the wet areas so we need a strategy to discriminate between wet and dry areas in the numerical simulation. In the SW context, this is usually called the wetting and drying (WD) problem. In our previous work $[3$, we showed the advantages of modifying the speed of waves near the vacuum region based on Toro's approach 4 to mitigate stability concerns. However, this still leads to the formation of a non-physical thin layer in the numerical solution (see figure 1 for an illustration). This unphysical thin layer could extend large distances from the realistic main body of the flow, which can cause inaccurate construction of the boundary, loss of conservation or severe numerical instabilities in the numerical solution. Besides the numerical issues,

25 determining probable flow extents through numerical simulation is critical for application of SW equations to geophysical flow. For example, in preparing a hazard map for a volcano or a flood, it is crucial to know the location of the front of the flow to answer basic questions such as - Does the flow reach a specific 


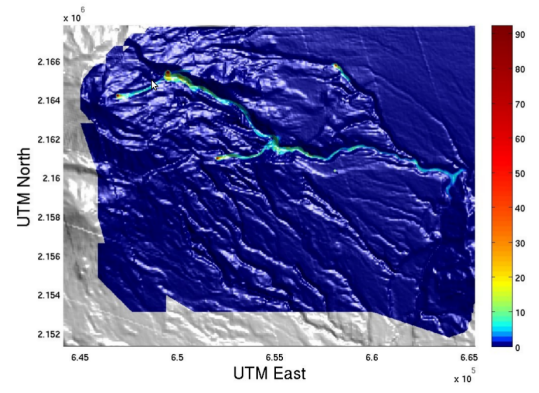

(a) Result without any control. A thin layer of flow covers most of the domain.

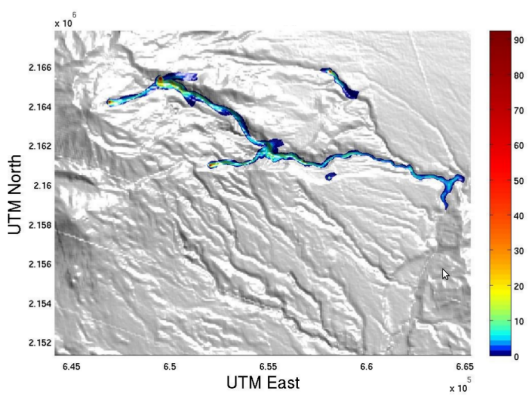

(b) Only flow with depth greater than $0.5 \mathrm{~m}$ is displayed.

Figure 1: Thin layer problem in maximum over time flow depth simulation of the 1955 debris flow at Atenquique, Mexico [5].

location? What is the distance of high risk locations from civil infrastructure?

30 The answer of all the above questions is not possible without good information about the interface of the flow along its flow path. A demonstration of possible issues in shown in Figure 1. This figure displays the numerical simulation of a block of ash flow in Atenquique, a village near the Colima volcano in Mexico, using a SW like model based on a granular flow assumption [3. The left figure shows the numerical solution of flow height without using any control for the thin layer problem and the right figure shows the same result using a naive control - plotting the regions with flow height $h>0.5 m$ (a threshold deemed too high for hazard analysis). As can be seen in the figure 1a, if no control on the numerical solution is used a thin layer of the flow covers a huge part of

40 the domain which causes instability and inaccuracy in the obtained results. To summarize, the following major difficulties arise in numerical solutions of SW flows related to the WD or thin layer problem:

1. Ambiguous and subjective computation of flow spreads.

2. Unphysical fast estimate of the flow speed. The state variables in the SW equations are momentum, $\left\{h V_{x}, h V_{y}\right\}$ (product of flow height and 
velocity). To find flow velocities, $\left\{V_{x}, V_{y}\right\}$, used during the solution process the momentum (often a small number) must be divided by flow depth, $h$ (also small near the flow boundary), which can causes a large numerical error and results in overly large flow velocities.

3. Unphysical thin layer (orders of magnitude thinner than a grain of sand). This results from unphysical speeds and the numerical wicking away of material.

4. Loss of numerical stability. Wave-speeds can become infinite at the flow boundary which means that flow equations lose their hyperbolicity at the vacuum state interface.

In addition to scaling issues, correctly identifying interface regions will allow us to construct models for other interesting physics (e.g. entrainment) that happens at the interface.

\subsection{Background and Review}

A review of the literature shows numerous attempts have been made to overcome these problems $[6,7,8,9,10,11,12,13$. Here we briefly review the problem and recent approaches and refer the interested reader to the references for more information. Generally, all of the approaches can be categorized into two basic groups which we name heuristic methods and interface tracking/capturing ${ }_{65}$ methods. The basic idea of heuristic methods [8, 9, 13, 11] is to reconstruct the flow interface using a simple heuristic or simple algebraic relations. In this approach, the reconstruction may take into account the physics of the problem, but this is not required. For example, to mitigate the thin layer problem a threshold could be defined to control the thin layer, the entire domain could be filled with 70 a shallow level of fluid (i.e. no interface), the flow could be reconstructed using a threshold, or the flux could be adjusted to avoid "thin" flows. It is possible to use any combination of these heuristic fixes. In interface tracking/capturing approaches an auxiliary set of equations is coupled to the original problem and used to follow the flow interface over time. The particular choice of the auxiliary

75 equations leads to either interface tracking (Lagrangian) or interface capturing 
(Eulerian) methods. In Lagrangian methods, the front is replaced with a set of points which explicitly define the location of the interface. During each time step these points move due to the numerically computed velocity field. Marker And Cell (MAC) [14, Simplified Marker And Cell (SMAC) [15] and the Surface Marker [16] methods are some examples of Lagrangian interface tracking techniques. The accuracy of the method is highly dependent on the number of particles that form the boundary. High computational cost, a tendency to form numerical instabilities and the inability to track complex topological changes are the significant drawbacks of Lagrangian techniques [17, 18, 19, 20, 21].

In the current paper, we focus on interface capturing or Eulerian methods. In these methods, the interface is implicitly specified by an additional scalar field defined in the entire computational domain. This additional field is coupled to the other governing equations and evolves due to the underlying fluid flow field. The level set, phase field and Volume of Fluid (VOF) methods are 90 popular instances of implicit/Eulerian methods. Hirt and Nichols [21] were the first to propose a VOF method. In this method, the scalar variable is the fraction/volume of a particular fluid in each cell. To construct the interface based on this method, an interface reconstruction technique is performed at each time step. Youngs 22 achieved a significant improvement in the VOF by adding a 95 piecewise linear interface (PLIC) representation of the fluid boundary. Youngs' method has been shown to be robust and efficient, but it is only a first order scheme. More advanced VOF methods are also available in Gerlach [23] and Gopala and van Wachem 24], but these methods require more complicated logic to reconstruct the interface. The phase field method is an Eulerian interface capturing scheme where the interface is implicitly related to an order parameter which shows the phase variation on the domain [20. In this method, the interface is a well-defined diffusive region between phases. The mass conserving Cahn-Hilliard and non-conservative Allen-Cahn formulations are two principal formulations of this method [25, 26, 27]. Mathematically, the conservative form requires a numerically challenging fourth order derivative while the other one has only second order derivatives. To date, no published work has used the 
phase field method to capture an interface in the shallow water equations. The Level Set method introduced by Osher and Sethian [19] is another Eulerian interface capturing technique. In this method, the scalar variable is typically a signed distance function which indicates the distance of a grid point to the interface. Quecedo and Pastor [28] is the only instance we have found of a level set based approach to mitigate the wetting-drying problem in the context of their Taylor-Galerkin approach to solve the SW equations. They presented two different options for handling the wetting-drying areas - a "simple yet efficient" method which is heuristic method in our classification and a level set based approach. Although they claimed that they used the level set as the second approach, no results using the method were included in the article and concluded that the level set is expensive and unnecessary for their problem.

\subsection{Contributions and Summary of Methodologies Used}

In this paper, we describe an approach to the thin layer issue (problems 1-4) discussed in section 1 in two parts. The first part is to identify the interface of the flow and the next is to make suitable modifications to meshes, state variables and fluxes based on the results of the first part. To identify the interface we study three different methods. The first one is a new multifaceted heuristic approach that uses a very small dimensionless threshold, which we call it GEOFLOW_TINY, to distinguish between wet and dry areas. The other methods of interface capturing that we implement are the phase field and level set approaches. To the best of our knowledge, the two latter methods are for the first time being applied to geophysical flow.

As there are different types of level set and phase field methods available in the literature, we initially use the standard level set method and the AllenCahn description of the phase field, and modify them as necessary for our problem. The details of implementation of all three methods are carefully explained. In the heuristic approach, after finding the wet and dry cells by using GEOFLOW_TINY, the cells are divided into three groups: wet, dry and partially wet cells. Next we adjust the fluxes for the partially wet cells and then finally 
update the state variables for wet cells. In the level set and phase field approaches, refining close to cells to the identified interface enables us to control the thin layer problem. We compare the numerical results to experiments of a granular SW flow over an inclined plane ending in a horizontal surface and field data from the Colima volcano.

The results show that all of the methods can address the thin layer problem and other related issues. Moreover, they indicate higher accuracy can be achieved by using the interface capturing methods though the computational cost may be higher. To decrease the computational cost, we used different techniques. For example in the phase field method, we used the Allen-Cahn formulation which is computationally less expensive compared to the CahnHilliard formulation, but it is not mass conservative. To preserve the mass, we have included a Lagrange multiplier based approach [29, 27. To decrease the computational cost further, we have used an operator splitting for time integration. Another advantage of the interface capturing methods is that they have stronger mathematical and physical structure, and more complicated physics can be coupled with the conservation equations. The heuristic methods are simple and robust and work well when properly tuned but our experience shows that finding a good threshold in the heuristic method that is appropriate for all problems is not easy. Finally, although we have implemented these methods for dry granular SW flows the methods themselves are applicable for other types of SW flows.

The structure of the rest of this paper is as follows. In the next section the shallow-water equations for geophysical flows is described. After that the common features of the solver that we used for all of the three methods are introduced. In section 4 , the different methods that were employed to mitigate the wetting-drying problem are explained, and finally discussion and conclusions complete the presentation. 


\section{Governing Equations}

The Savage-Hutter equation for geophysical flows was first introduced in the late 1980's. The original model has subsequently been improved upon by SavageHutter themselves as well as others [30, 31, 32, 33, 34, 35. In our earlier work [36, 3, 37, we developed the TITAN2D depth-averaged geophysical flow simu-

$$
\frac{\partial}{\partial t}\left\{\begin{array}{c}
h \\
h v_{x} \\
h v_{y}
\end{array}\right\}+\frac{\partial}{\partial x}\left\{\begin{array}{c}
h v_{x} \\
h v_{x}^{2}+\frac{k_{a p}}{2} g_{z} h^{2} \\
h v_{x} v_{y}
\end{array}\right\}+\frac{\partial}{\partial y}\left\{\begin{array}{c}
h v_{y} \\
h v_{x} v_{y} \\
h v_{y}^{2}+\frac{k_{a p}}{2} g_{z} h^{2}
\end{array}\right\}=\left\{\begin{array}{c}
S_{h} \\
S_{x} \\
S_{y}
\end{array}\right\},
$$

where:

$$
\begin{aligned}
S_{x} & =g_{x} h-\frac{V_{x} \tan \left(\phi_{b e d}\right)}{\sqrt{V_{x}^{2}+V_{y}^{2}}}\left(g_{z} h+\frac{h V_{x}^{2}}{r_{x}}\right)-h k_{a p} \operatorname{sgn}\left(\frac{\partial V_{x}}{\partial y}\right) \frac{\partial\left(g_{z} h\right)}{\partial y} \sin \left(\phi_{\text {int }}\right), \\
S_{y} & =g_{y} h-\frac{V_{y} \tan \left(\phi_{b e d}\right)}{\sqrt{V_{x}^{2}+V_{y}^{2}}}\left(g_{z} h+\frac{h V_{y}^{2}}{r_{y}}\right)-h k_{a p} \operatorname{sgn}\left(\frac{\partial V_{y}}{\partial x}\right) \frac{\partial\left(g_{z} h\right)}{\partial x} \sin \left(\phi_{\text {int }}\right), \\
k_{a p} & =2 \frac{1 \pm\left[1-\cos ^{2} \phi_{\text {int }}\left(1+\tan ^{2} \phi_{\text {bed }}\right)\right]^{\frac{1}{2}}}{\cos ^{2} \phi_{\text {int }}}-1,
\end{aligned}
$$

in $k_{a p},-$ corresponds to an active state $\left(\frac{\partial \bar{v}_{x}}{\partial x}+\frac{\partial \bar{v}_{y}}{\partial y}>0\right)$, and + corresponds to a passive state $\left(\frac{\partial \bar{v}_{x}}{\partial x}+\frac{\partial \bar{v}_{y}}{\partial y}<0\right)$.

In the above equations, the coordinate system (see Figure 2 is aligned such that $x$ and $y$ are directions tangential to the surface of the $3 \mathrm{D}$ terrain and $z$ is normal to the surface. $h$ is the flow depth in the $z$ direction. $h V_{x}$ and $h V_{y}$ 

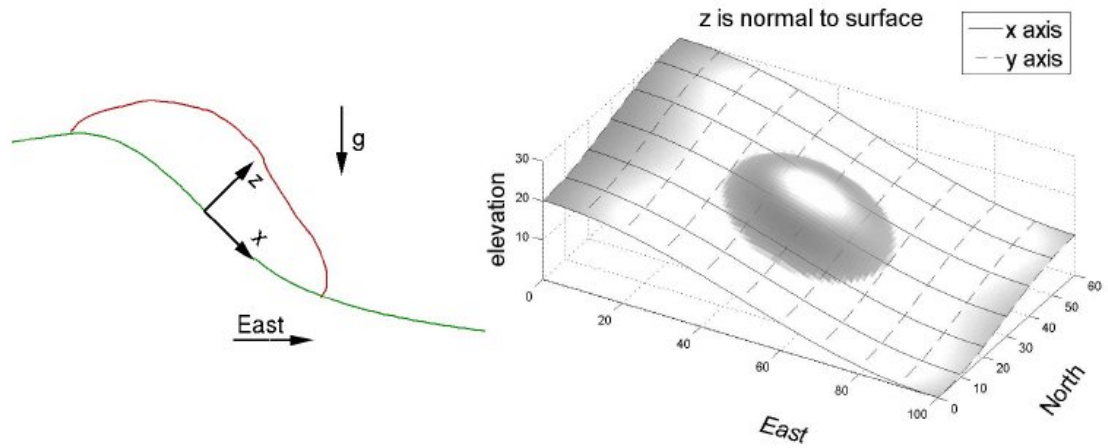

Figure 2: In the local coordinate system the $z$ direction is normal to the surface; the $x$ and $y$ directions are tangential to the surface.

are the components of "momentum" in $x$ and $y$ directions, respectively, and the effect of terrain elevation changes is represented by gravitational source terms.

Note that in the momentum equations $k_{a p} g_{z} \frac{h^{2}}{2}$ is the contribution of hydrostatic pressure to the momentum fluxes. $S_{h}$ is a source of mass, i.e. material that either effuses or erodes out of the ground. $S_{x}$ is the sum of a gravitational driving force, the friction that resists motion of the material relative to the bed, and the friction that resists the internal shearing motion of the material:

TITAN2D solves the above system of equations using a finite volume Godunov method:

$$
U_{i}^{n+1}=U_{i}^{n}-\frac{\triangle t}{\triangle x}\left\{F_{i+\frac{1}{2}}^{n}-F_{i-\frac{1}{2}}^{n}\right\}-\frac{\triangle t}{\triangle y}\left\{G_{i+\frac{1}{2}}^{n}-G_{i-\frac{1}{2}}^{n}\right\} .
$$

In the above equation, $\mathrm{G}$ and $\mathrm{F}$ are the flux terms at the inter-cell boundaries which are computed by the Harten-Lax-Van Leer (HLL) [38] Riemann solver that is explained in the next section.

\section{SW Solution and Adaptive Strategy for WD Interface}

\subsection{Using Appropriate Riemann Fluxes}

Following Toro 4, TITAN2D uses the HLL Riemann average of fluxes. Let $U$ be a state variable, $F$ be a flux of that state variable, $s$ be a maximum 
wave speed, and $R$ and $L$ subscripts which denote "right" and "left" values respectively. The HLL Riemann flux is then

$$
F_{H L L}= \begin{cases}F_{L} & s_{L} \geq 0 \\ F_{R} & s_{R} \leq 0 \\ \frac{s_{R} F_{L}-s_{L} F_{R}+s_{L} s_{R}\left(U_{R}-U_{L}\right)}{s_{R}-s_{L}} & \text { otherwise }\end{cases}
$$

195 


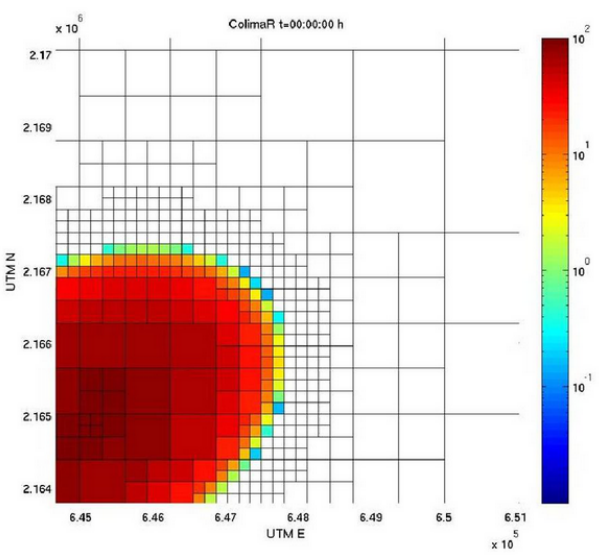

Figure 3: This figure shows four layers of the maximally refined elements near the interface that we call them buffer layer. Creating buffer layers is one of the key strategies that we do to control the thin layer problem in all of the methods that we studied in this work.

"rings" of maximally refined buffer cells. The number of rings are equal to or greater than the number of iterations between mesh adaptations. We usually adapt the mesh after every 5 time steps. This strategy limits artificially high transportation of material from the inside of the interface to outside of the interface and guarantees that material will only flow into maximally refined dry cells at the wet-dry front. However, some material that is outside of the recognized boundary may be left outside the outermost band of buffer cells. These cells are not updated until the interface passes them. The rings of buffer cells strategy decreases the numerical wicking problem and when combined with interface capturing scheme it is sufficient to decrease other thin layer problems to a level that prevents the loss of stability.

Clearly this strategy depends on the way that we describe the interface. In the heuristic approach, we use flow below GEOFLOW_TINY for selecting the buffer layers. This threshold does not exactly show the interface, and our experience shows that it depends on the scale of the flow and may vary for different DEMs; therefore, we select 4 buffer layers each composed of 5 rings of refined cells at $h$ equal to $1,5,15$ and 40 times of the GEOFLOW_TINY. In the level set and 
the phase field methods we know the location of the interface at the time of computation. Thus we just make 2 buffer layers one exactly before and the other after the interface, each of them having 5 rings of refined cells. Knowing the interface location allows us to decrease the number of buffer layers from 4 to to unrefinement.

\section{Interface Capturing Strategies}

We have now reviewed the major difficulties and solution approaches for the WD problem in the solution of shallow water equations. For added motivation,

computations for the interface capturing schemes. Unrefinement takes place immediately after refinement; a group of four "sibling" cells will be selected to merge into their mutual "parent" cell if the sum of their inter-cellular fluxes is very low compared to the average flux. Cells that have either just been refined or are otherwise in any of the current bands of buffer cells are immune the result of a simulation for Atenquique debris is displayed in figure 1. The left picture is the obtained result from the solver and the right picture is the same result, but flow height is displayed only for flow height greater than $0.5 \mathrm{~m}$. This picture shows that without correction the dominant part of the domain is filled with a thin layer that makes it difficult to identify the actual WD interface. numerical difficulties of SWE introduced above are detailed.

\subsection{Heuristic methods}

Usually, heuristic methods use one or a combination of the following four strategies [6]:

1. filling the entire computational domain with a thin layer of fluid;

2. using a threshold to check whether a cell or a node is wet or dry (or possibly partially wet), and then making a decision to add or remove it from the computational domain; 


\begin{tabular}{|l|l|l|}
\hline Strategy & Mass Conservation & Physics \\
\hline Thin film & $\begin{array}{l}\text { Adequate, but requires } \\
\text { solution reconstruction }\end{array}$ & $\begin{array}{l}\text { Produces a smooth and } \\
\text { realistic wetting front }\end{array}$ \\
\hline Cell removal & $\begin{array}{l}\text { Dependent on numeri- } \\
\text { cal method for solving } \\
\text { the equations }\end{array}$ & $\begin{array}{l}\text { Excellent, performs } \\
\text { better on advancing } \\
\text { front than receding } \\
\text { front }\end{array}$ \\
\hline VFR & $\begin{array}{l}\text { Conservative, with aid } \\
\text { of some correction pro- } \\
\text { cedure }\end{array}$ & $\begin{array}{l}\text { Very good in wide ver- } \\
\text { ity of problems }\end{array}$ \\
\hline $\begin{array}{l}\text { Allowable negative } \\
\text { depth }\end{array}$ & $\begin{array}{l}\text { Conservative, but per- } \\
\text { formance depend on } \\
\text { WD parameters }\end{array}$ & $\begin{array}{l}\text { Same as mass conserva- } \\
\text { tion }\end{array}$ \\
\hline
\end{tabular}

Note that the heuristic method we use in this study can be categorized as a VFR method.

\subsubsection{Selecting an appropriate threshold}

In this approach, we use a very small threshold of a scaled flow height to segregate the wet, partially wet and dry cells. This identification allows other schemes like those for flux adjustment and boundary reconstruction to identify where the flow is non-physically thin, i.e. where should they consider the boundary of the flow to be. Consistency is the key requirement of whatever strategy we choose to determine the scaling. That is, the chosen strategy must be able to 
generate the same "appropriate" value for depth scale at the beginning and at the end of a simulation. In a "typical" geological simulation, say of the collapse of a volcanic dome which would be modeled in TITAN2D as a "pile source", the initial body of mass can be quite deep, but at the end of the simulation the material will likely be spread over a large area. Hypothetically, if one were to scale by maximum flow depth at the beginning and end of the simulation, they would obtain vastly different values. More importantly, if the only source of mass was an effusion of material out of the ground, the maximum initial flow depth would be zero, even if the total volume during the course of the simulation was the same as the previously mentioned "pile source". On the other hand, scaling by the cube root of the total volume of the flow being simulated is entirely consistent and is the flow depth scaling factor used by TITAN2D. We do not claim that the cube root of volume is any more or less appropriate as a scaling factor than maximum initial flow depth commonly used in other shallow water contexts, for example storm surge simulation. Having chosen a consistent scaling factor we were then able to define associated non-dimensional depths for negligibly thin and merely thin flows. If one were to assume that a particular geophysical mass flow event involved a volume of $10^{8}\left[\mathrm{~m}^{3}\right]$, it would then be reasonable to state that flow depths of less than $5[\mathrm{~cm}]$ were both negligible and non-threatening. This roughly equates to non-dimensional negligible flow depth, which we call GEOFLOW_TINY, with the value

$$
\text { GEOFLOW_TINY }=0.0001 .
$$

275 47 48 49 50 51 52 53 54 55 56 57 58 59 60 61 62 63 
In addition, the familiar continuum equations loose hyperbolicity (wave speeds tend to infinity) at the boundary between a continuous material and a vacuum. Having implicitly defined the flow boundary, we prevent calculation of state variables outside the flow, i.e. in regions with flow depth below GEOFLOW_TINY. This reduces both non-physical flow spreading and computational cost. Note that state variables in cells with flow depth less than GEOFLOW_TINY are not zeroed.

\subsubsection{Interface Reconstruction}

As noted above, the use of a standard Eulerian grid imposes discrete fixed increments to the flow extent which contributes to the wicking problem (Problem 2 ) at the boundary. Use of adaptive mesh refinement reduces this error by reducing the amount of the increment. The Lagrangian approach does not have this limitation. Tai et al. [40] took advantage of when they augmented their one-dimensional NOC scheme with Lagrangian front tracking. However, implementing a hybrid Eulerian-Lagrangian scheme in two dimensions is significantly more complex. Therefore, we implemented a very simple and inexpensive interface reconstruction and predictive Lagrangian front tracking scheme. Knowledge of the interface allows us to generate a representative average for an individual cell edge over the time-step and for values of state variables which are then used to compute inter-cellular fluxes into/out-of partially wet cells. The interface reconstruction scheme is illustrated in figure 4. Specifically, each partially wet cell is assumed to be split by a straight line into a completely dry and a completely wet part. For the sake of simplicity, we restrict this line to one of four orientations: east-west, north-south, or parallel to either diagonal of the square cell. However, all placements/translations of the wet-dry line are allowed. At the beginning of each time-step, the orientation of the line for the entire time-step is set based solely on which of the cell's neighbors have 

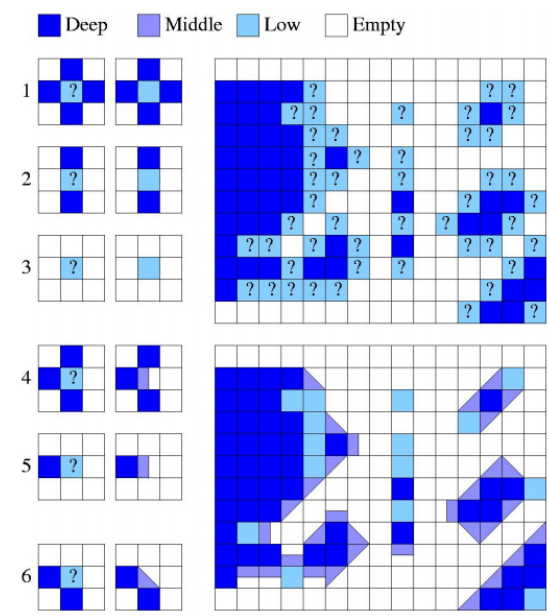

Figure 4: Interface Reconstruction in TITAN2D.

flow depth greater/less than the GEOFLOW_TINY threshold 1 . Orientation of the wet-dry line, and which side of it is wet, is indicated by a single integer representing the geometrically determined "most wet node." The only nine possible values for the most wet node are any of the cell's corners, edge midpoints or its center. The most wet node is assumed to have a flow depth that is the maximum of the cell or any of its four neighbors. The placement of the line is such that the volume of material in the cell under a plane passing through the most wet node and the wet dry-line is the same as the unadjusted cell. Since we now know where the wet-dry line is at the beginning of the time step, we can compute which of the cell's edges are completely wet, completely dry, or partially wet, and the fraction of wetness for the partially wet edges. Given the orientation and beginning of time-step location of the wet-dry line and the cell's state variables, it is also fairly straightforward to predict the line's end of time-step location. This is done by convecting the wet-dry line at the shock speed, $s=v+a$ where the speed of sound has been adjusted to account for

\footnotetext{
${ }^{1}$ As stated in Subsection 3.2 the refinement strategy ensures that the flow front will always be maximally refined. This simplifies the coding since all cells at the front can be safely assumed to have only one neighbor on each side.
} 
only part of the cell being wet, i.e. $a=\sqrt{k_{a p} g h \frac{A}{A_{w e t}}}$. Here $A$ is the whole cell's area and $A_{w e t}$ is the portion of the cell's area that is wet. Since the wet-dry boundary within each partially wet cell is assumed to be a straight line with (during the time-step) fixed orientation, convecting a single point, in this case, the midpoint on the line, is equivalent to convecting the entire wet-dry line. A spatial and time average of the wetness factor for the edge over the time-step can therefore be easily computed as

$$
W=\left(\frac{0 \cdot \Delta t_{d r y}+\frac{1}{2}\left(w_{\text {beg }}+w_{\text {end }}\right) \Delta t_{\text {part }}+1 \cdot \Delta t_{\text {wet }}}{\Delta t}\right)\left(\frac{A}{A_{\text {wet }}}\right)
$$

where $\Delta t=\Delta t_{d r y}+\Delta t_{\text {part }}+\Delta t_{\text {wet }}$ is the entire time-step, $\Delta t_{d r y}$ is the portion of the time-step for which the edge is completely dry, $\Delta t_{\text {part }}$ is the portion of the time-step for which the edge is partially wet and partially dry, $\Delta t_{w e t}$ is the portion of the time-step for which the edge is completely wet, $w_{\text {beg }}$ is the edge's fraction of wetness at the beginning of the time-step, and $w_{\text {end }}$ is the edge's fraction of wetness at the end of the time-step. As explained above the wet, dry and partially wet part of the cell can be computed. Given this information, we do not actually need to calculate the amount of time that a cell is partially wet, completely dry, and completely wet. Instead, when it starts dry and ends wet, we calculate the wetness factor. The wet-dry line has an initial orientation and a "one-dimensional" position measured from the wettest node to the wet dry line. Since, we know that it is convected with constant wave speed during the updating scheme, we can calculate the point in time at which each node changes from wet to dry (or vice versa). Consequently, the amount of time that a cell edge is completely dry is the amount of time that both of its end points are dry and the amount of time it is completely wet is the amount of time that both endpoints are wet.

\subsubsection{Adjusting Fluxes in Partially Wet Cells}

The state variables used to compute the physical fluxes are the whole cell average values multiplied by the edge wetness factor. Note this results in the zeroing of this cell's physical fluxes for its sides that will be completely dry for the 
entire time-step and increasing them for sides that will be completely wet for the entire time-step. The numerical fluxes are then taken to be the HLL Riemann average of the "adjusted" physical fluxes from the cells on both sides the edge. The wetness factor adjustment of fluxes delays/decreases the transfer of material from partially wet cells to completely dry cells. This delay significantly reduces the amount of non-physical flow spreading, and requires negligible additional computation and memory usage. In terms of memory usage, this scheme only requires one additional integer indicating the most wet node, and two additional decimal numbers for the cell's fraction of wet area and the location of the wet-dry line's midpoint (implemented as a single number ranging from zero to one). The flux adjustment in partially wet cells mitigates the numerical wicking (Problem 2) at the boundary but not within the flow (that requires either a uniform grid or an adaptive strategy based on fluxes and the rings of buffer cells strategy).

\subsection{Second: Phase Field Method}

In this method, the state variables are augmented by a continuous order parameter. This order parameter, $\varphi$, implicitly represents the interface in the domain. To this aim, a new transfer equation must be solved which is coupled with the other state variables. Papers [20, 41, 42, 43, are good references about the history and evolution of the method. Phase diffusion methods and particularly the phase field method is based upon the notion that the interface between phases is a diffusive region rather than a sharp interface. The value of $\phi$ is constant within a bulk phase but changes smoothly between the phases. In this work $\phi$ is 1 for the fluid phase $\{\mathbf{x}: \phi(\mathbf{x}, t)=1\}$, and it is -1 for void regions $\{\mathbf{x}: \phi(\mathbf{x}, t)=-1\}$, and is between -1 to 1 on the diffusion region $\{\mathbf{x}:-1<\phi(\mathbf{x}, t)<1\}$. We can implicitly assume the interface of the flow is where $\{\mathbf{x}: \phi(\mathbf{x}, t)=0\}$. In this study, we have used the Allen-Cahn form of the phase field.

$$
\frac{\partial \varphi}{\partial t}+\vec{V} \cdot \nabla \varphi=\gamma\left(\triangle \varphi-F^{\prime}(\varphi)\right)
$$


where:

$$
F(\varphi)=\frac{1}{4 \eta^{2}}\left(\varphi^{2}-1\right)^{2}, \text { so } \quad F^{\prime}(\varphi)=\frac{\delta F}{\delta \varphi}=\frac{1}{\eta^{2}} \varphi\left(\varphi^{2}-1\right) .
$$

In the above equation, $\eta$ is a constant that regulates the capillary width or diffusion width, $\gamma$ denotes an elastic relaxation constant, and $F(\varphi)$ is a mixing free energy which is the well-known double-well potential function and represents the interactions of different volume fractions of individual species [44, 45].

This formulation is easier to solve, but is not mass conservative. To conserve the mass, we follow [29, 27, and use a Lagrange multiplier $\xi(t)$ modified as $\left(\varphi^{2}-1\right) \xi(t)$ to enforce the depth averaged conservation of form of the mass $\frac{D}{D t} \int_{\Omega} \varphi h d x=0$. This results in an additional source term in (6). The $\left(\varphi^{2}-1\right)$ modification for the Lagrange multiplier minimizes the change $\varphi$ on the bulk region i.e when $\varphi= \pm 1$. The final form of equation (6) and the constraint are:

$$
\begin{aligned}
& \frac{\partial \varphi}{\partial t}+\vec{V} \cdot \nabla \varphi=\gamma\left(\Delta \varphi-F^{\prime}(\varphi)+\left(\varphi^{2}-1\right) \xi(t)\right), \\
& \frac{D}{D t} \int_{\Omega} h \varphi d x=0
\end{aligned}
$$

Physically (9) implies that the volume of pile must be constant during the simulation. A simple relationship for $\xi(t)$ is derived as follows:

$$
\begin{aligned}
\frac{D}{D t} \int_{\Omega} \varphi h d x & =h \int_{\Omega} \frac{D}{D t} \varphi d x+\underbrace{\varphi \int_{\Omega} \frac{D}{D t} h d x}_{=0 \text { continuity }} \\
& =h \int_{\Omega} \gamma\left(\triangle \varphi-F^{\prime}(\varphi)+\varphi\left(\varphi^{2}-1\right) \xi(t)\right) \quad d x=0,
\end{aligned}
$$

The first term in above relation will be neglected by applying the Gauss' theorem for the Neuman boundary conditions $\left(\left.\frac{\partial \varphi}{\partial n}\right|_{\Gamma}=0,\right)$ :

$$
\int_{\Omega} h \gamma \nabla \cdot \nabla \varphi d x=\int_{\Gamma} h \gamma \nabla \varphi d s=0,
$$

so to always satisfy the constrain $(9)$ we end up with:

$$
\frac{1}{\eta^{2}} \int_{\Omega} \varphi\left(\varphi^{2}-1\right) d x=\xi(t) \int_{\Omega}\left(\varphi^{2}-1\right) d x \Rightarrow \xi(t)=\frac{\int_{\Omega} \varphi\left(\varphi^{2}-1\right) d x}{\eta^{2} \int_{\Omega}\left(\varphi^{2}-1\right) d x}
$$

330

We set $\eta=n \delta x$, where $\delta x$ is equal to the smallest cell length of all elements and $n$ is the number of the buffer layers explained in section 3.2 For time 
integration of equation (8) we use operator splitting. The Euler explicit method is used for time integration of all terms except the Laplacian terms, which are updated implicitly. For a stable explicit time integrator, the size of the time step has to satisfy the CFL condition.

$$
\begin{aligned}
\frac{\varphi^{i+.5}-\varphi^{i}}{\triangle t} & =\gamma\left(-F^{\prime}\left(\varphi^{i}\right)+\varphi^{i}\left(1-\left(\varphi^{i}\right)^{2}\right) \xi\left(t^{i}\right)\right) \\
\frac{\varphi^{i+1}-\varphi^{i+.5}}{\triangle t} & =\gamma \nabla \cdot \nabla \varphi^{i+1}
\end{aligned}
$$

We used GMRES solver of the PETSc [46] library to solve equation (14). Since the Krylov subspace solvers just needs the result of matrix-vector multiplication, we used matrix-free method to compute the Laplacian term to decrease the memory cost of implicit solver.

\subsection{Third: Level Set Method}

The Level Set method is another Eulerian interface capturing method, originally introduced by Osher and Sethian [19]. There are many papers about Level Sets for interface capturing in flow simulations [47, 48, 49, but the application of the method is not limited to these [50, 51]. The basis of the method is to capture the interface by solving a hyperbolic Hamilton-Jacobi PDE on the computational domain for $\Psi(X, t)$, a signed distance function which is zero on the interface and has an absolute value in the domain depending on the distance to the zero level. The level set variable $\Psi(X, t)$ implicitly represents the interface. $\Psi(X, t)$ is positive outside the interface and negative inside. Given the initial condition $\Psi_{0}(X, t)$ the advection of the interface $(\Psi(X, t)=0)$ only depends on the normal velocity $F$ :

$$
\frac{\partial \Psi}{\partial t}+F|\nabla \Psi|=0
$$

substituting $F=\vec{V} \cdot \vec{n}$ which $\vec{n}$ is the normal vector, and is equal to $\frac{\nabla \Psi}{|\nabla \Psi|}$ leads to:

$$
\frac{\partial \Psi}{\partial t}+\vec{V} \nabla \Psi=0
$$




\subsubsection{Reinitialization}

The solution of equation 16 might not remain a signed distance function. To keep $\Psi$ a signed distance function, we need a procedure that is called reinitialization. There are several techniques for this purpose [19, but what we implemented here is a composition of the method presented in Chopp's work [52] with some modifications for the points close to the interface, and a PDE based reinitialization for the further places [53]. PDE based reinitialization is easy for parallelization, though it has been known to induce mass loss. Chopp suggests a bicubic interpolation for the points near the interface [52], but here we use a bilinear interpolation which is not as accurate, but it is accurate enough to preserve the interface and cheaper. For points far from the interface we do not need a very accurate $\Psi$, so we use a PDE based reinitialization which is easier to implement and has lower computational cost. In our approach, we first find $\Psi$ for the points that are adjacent to the interface, then use these points to update the signed distance function for rest of the domain using a PDE based method. In this way, the interface $(\Psi=0)$ is preserved with an affordable computational cost and effort. Moreover, since just $\Psi$ is required for the points that are located near the interface, there is no need to update it for the whole domain. So in this work we select $\Psi_{\text {thresh }}<10 \delta x$, where $\delta x$ is the smallest size of the elements, as the threshold for updating $\Psi$ in the PDE-based reinitialization process. The signed distance function is updated for points adjacent to the interface in the following steps:

1. First we find the adjacent points to the interface, and store them in a list that is usually called the accepted point list in the literature [52].

2. The next step is to determine the interpolant for the level set function, $p(X)$, that returns $\Psi(X)$ for a point $X$.

3. The last step is to compute the distance of the accepted points based on the following conditions:

$$
\begin{aligned}
& p\left(X_{\text {int }}\right)=0, \\
& \nabla p(y) \times\left(X_{0}-X_{\text {int }}\right)=0,
\end{aligned}
$$


where $X_{i n t}$ is the nearest point on the interface, and $X_{0}$ is the point in the accepted list that we want to compute its distance to the interface. The first condition 17a requires that $X_{i n t}$ is on the interface, and the second condition states that the interface normal at the closest point must pass through $X_{0}$. In this part, we followed the notation of Chopp's paper [52].

In the first step, we just need to find those points where the sign of $\Psi$ is different from their neighbors, and store them in a list that we call them accepted points list. For the second step, there are different ways to perform the interpolation. In many applications, like in this study, the initial interface has a specific geometrical shape such as a circle or ellipse, so we do not need to find this interpolant approximately, and its equation can be used directly as $p(X)$. For example, in this study the initial pile has an elliptic shape, so the initial interface follows the elliptic equation. However, we will still have to use approximate $p(X)$ for reinitialization as the curve evolves.

If the equation of the interface is not known, which is usually the case, approximating the interface by interpolation is necessary. Chopp suggested a bicubic approximation [52] to achieve a fully second order accurate result, but this method requires the solution of a 16 by 16 system of equations for cells containing the interface to determine the coefficients of the bicubic interpolation. The purpose of the interpolation based method is to ensure that the interface does not undergo spurious motion. Therefore, the use of bilinear interpolation should be sufficient. The general form of $p$ is:

$$
p=a_{0}+a_{1} x+a_{2} y+a_{3} x y .
$$

A general way to find $a_{0}$ to $a_{3}$ for a grid with different element sizes is to solvuse the value of $\Psi$ at cell centers of four adjoining cells (see fig. 5).

$$
\left[\begin{array}{llll}
1 & x_{1} & y_{1} & x_{1} y_{1} \\
1 & x_{2} & y_{2} & x_{2} y_{2} \\
1 & x_{3} & y_{3} & x_{3} y_{3} \\
1 & x_{4} & y_{4} & x_{4} y_{4}
\end{array}\right]\left[\begin{array}{l}
a_{0} \\
a_{1} \\
a_{2} \\
a_{3}
\end{array}\right]=\left[\begin{array}{l}
\Psi_{1} \\
\Psi_{2} \\
\Psi_{3} \\
\Psi_{4}
\end{array}\right]
$$


In our case the elements near the interface are of uniform size, and the four cell centers can be considered as the vertices of a rectangle, so the coefficients of equation 18 can be computed as following:

$$
\begin{aligned}
a_{0}= & \frac{\left(\Psi_{1} x_{2} y_{2}-\Psi_{2} x_{2} y_{1}-\Psi_{3} x_{1} y_{2}+\Psi_{4} x_{1} y_{1}\right)}{\left(x_{2}-x_{1}\right)\left(y_{2}-y_{1}\right)} \\
a_{1} & =\frac{\left(-\Psi_{1} y_{2}+\Psi_{2} y_{1}+\Psi_{3} y_{2}-\Psi_{4} y_{1}\right)}{\left(x_{2}-x_{1}\right)\left(y_{2}-y_{1}\right)} \\
a_{2} & =\frac{\left(-\Psi_{1} x_{2}+\Psi_{2} x_{2}+\Psi_{3} x_{1}-\Psi_{4} x_{1}\right)}{\left(x_{2}-x_{1}\right)\left(y_{2}-y_{1}\right)} \\
a_{4} & =\frac{\left(\Psi_{1}-\Psi_{2}-\Psi_{3}+\Psi_{4}\right)}{\left(x_{2}-x_{1}\right)\left(y_{2}-y_{1}\right)}
\end{aligned}
$$

where $\Psi_{1}=\left(x_{1}, y_{1}\right), \Psi_{2}=\left(x_{1}, y_{2}\right), \Psi_{3}=\left(x_{2}, y_{1}\right)$ and $\Psi_{4}=\left(x_{2}, y_{2}\right)$ as can be seen in the figure 5 After finding the interpolation function $p$, we can compute

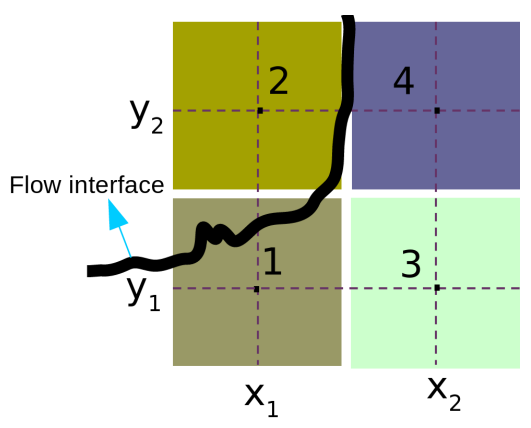

Figure 5: Configuration of the four elements that have been selected for bilinear interpolation. In this schematic example the interface passes through two hypothetical edges of (2-4) and (1-2) edges. This is just for demonstration purpose and has no effect on the equations.

the distance from a point to the interface using equations $17 \mathrm{a}$ and $17 \mathrm{~b})$. The 
solution is calculated using a variant of Newton's method as presented in [52]:

$$
\begin{aligned}
& \delta_{1}=-p\left(X^{k}\right) \frac{\nabla p\left(X^{k}\right)}{\nabla p\left(X^{k}\right) \cdot \nabla p\left(X^{k}\right)}, \\
& X_{1+\frac{1}{2}}=X_{k}+\delta_{1}, \\
& \delta_{2}=\left(X^{0}-X^{k}\right)-\frac{\left(X^{0}-X^{k}\right) \cdot \nabla p\left(X^{k}\right)}{\nabla p\left(X^{k}\right) \cdot \nabla p\left(X^{k}\right)} \nabla p\left(X^{k}\right), \\
& X_{k+1}=X_{1+\frac{1}{2}}+\delta_{2} .
\end{aligned}
$$

This is an iterative method that starts from $X_{0}$, which is the point whose distance to the interface must be determined. Once the nearest point on the interface is determined, the distance can be computed, and sign of $\Psi$ is the same as it was before reinitialization. For interpolation as shown in equation (18), 385 we need four points. When we create the accepted list, instead of storing single points we store a pair of points that are neighbors whose sign of $\Psi$ differs. Then to find two other points, it is enough to select a direction perpendicular to the direction that connects the selected pair, and find the neighbors of the first two points in this direction.

Before proceeding to the PDE-based step, we would like to make some remarks about the aforementioned step. In parallel computation, the domain is decomposed between multiple processors, with each processor holding a port responsible for a part of the domain. The information on neighboring processors is stored in "ghost"-cells, which are updated using inter-processor communication. In TITAN2D, only the neighboring ghost cells in the $x$ and $y$ directions are provided. Thus, a cell at location $(i, j)$ does not have direct access to the information stored at location $(i+1, j+1)$. This causes issues in the rare instances that the interface is near the corner of a processor's domain, figure 6 . To avoid excessive inter-processor communication in these rare cases, the bilinear interpolation described before is modified to purely linear interpolation method requiring only three points:

$$
p=a_{0}+a_{1} x+a_{2} y .
$$

When choosing the three points needed for interpolation, we ensure that only the 
points in Cartesian directions are used. With these three points the interpolant is given by:

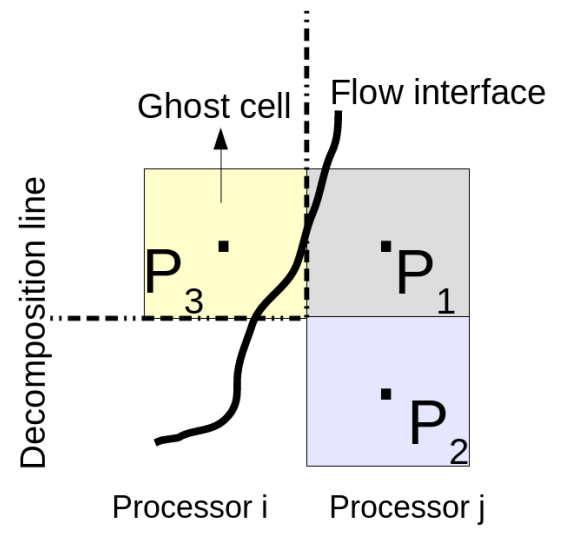

Figure 6: In this figure $p_{1}$ and $p_{2}$ are updated in processor $\mathrm{j}$ and $p_{3}$ is updated in processor $\mathrm{i}$, but its information is available in processor $\mathrm{j}$ as ghost cell.

$$
\begin{aligned}
& p_{1}=\left[\begin{array}{l}
x_{1} \\
y_{1} \\
\Psi_{1}
\end{array}\right], \quad p_{2}=\left[\begin{array}{l}
x_{2} \\
y_{2} \\
\Psi_{2}
\end{array}\right], \quad p_{3}=\left[\begin{array}{l}
x_{3} \\
y_{3} \\
\Psi_{3}
\end{array}\right], \\
& \vec{n}=\overrightarrow{p_{2} p_{1}} \times \overrightarrow{p_{3} p_{1}}=\left[\begin{array}{l}
n_{1} \\
n_{2} \\
n_{3}
\end{array}\right]=\left[\begin{array}{c}
\left(y_{2}-y_{1}\right)\left(\Psi_{3}-\Psi_{1}\right)-\left(y_{3}-y_{1}\right)\left(\Psi_{2}-\Psi_{1}\right) \\
\left(x_{3}-x_{1}\right)\left(\Psi_{2}-\Psi_{1}\right)-\left(x_{2}-x_{1}\right)\left(\Psi_{3}-\Psi_{1}\right) \\
\left(x_{2}-x_{1}\right)\left(y_{3}-y_{1}\right)-\left(x_{3}-x_{1}\right)\left(y_{2}-y_{1}\right)
\end{array}\right] \\
& a_{0}=\frac{n_{1}}{n_{3}} x_{1}+\frac{n_{2}}{n_{3}} y_{1}+\Psi_{1} \quad a_{1}=\frac{-n_{1}}{n_{3}}, \quad a_{2}=\frac{-n_{2}}{n_{3}}
\end{aligned}
$$

390 as the set container of the $\mathrm{C}++$ standard library, are useful data structures for storing the adjacent points near the interface. The reason for this is that this container keeps its item in a sorted way, and does not allow the storage of duplicate items. This simplifies the coding effort as we do not need manually 395 to check list every time that we want to save a new element.

After finding $\Psi$ for the points adjacent to the interface, $\Psi$ in other points 
can be updated with a PDE based reinitialization using an upwinding scheme. We use the following PDE for this aim:

$$
\frac{\partial \Psi}{\partial \tau}+\operatorname{sgn}\left(\Psi_{0}\right)(|\nabla \Psi|-1)=0 .
$$

In equation (25), $\tau$ is a pseudo-time and the equation should be solved until it converges reasonably. This $\operatorname{PDE}$ adjusts $\Psi$ such that $|\nabla \Psi|=1$. We solve equation (25) by the method introduced in [54]:

$$
\Psi_{i j}^{n+1}=\Psi_{i j}^{n}-\triangle t\left(\max (F, 0) \nabla_{i j}^{+}+\min (F, 0) \nabla_{i j}^{-}\right),
$$

where

$$
\begin{gathered}
\nabla_{i j}^{+}=\left[\max \left(D^{-x} \Psi_{i j}^{n}\right)^{2}+\min \left(D^{+x} \Psi_{i j}^{n}\right)^{2}\right. \\
\left.\max \left(D^{-y} \Psi_{i j}^{n}\right)^{2}+\min \left(D^{+y} \Psi_{i j}^{n}\right)^{2}\right]^{1 / 2} \\
\nabla_{i j}^{-}=\left[\min \left(D^{-x} \Psi_{i j}^{n}\right)^{2}+\max \left(D^{+x} \Psi_{i j k}^{n}\right)^{2}\right. \\
\left.\min \left(D^{-y} \Psi_{i j}^{n}\right)^{2}+\max \left(D^{+y} \Psi_{i j}^{n}\right)^{2}\right]^{1 / 2}
\end{gathered}
$$

In the above equations $D^{+}$, and $D^{-}$are the backward and forward differences, respectively, in the corresponding directions. We solve equation 25 until $\sum_{i j}\left|\Psi_{i j}^{n+1}-\Psi_{i j}^{n}\right|<.5(\delta x)^{3}$ over all points being updated.

\section{Results}

We use two cases to illustrate the interface capturing schemes. The first case is flow down an inclined plane while the second is past eruptions at the Colima Volcano. For the first case, we have access to detailed and accurate experimental data and careful comparisons are possible. The second is more characteristic of typical field observations available to calibrate and validate TITAN flows and only sparse deposit data is available. Since the computational cost of the methods is not the same, we have also compared these methods from a computational point of view by the required simulation time and the number of elements generated in the adaptive meshing process during the simulation. 


\subsection{Inclined plane}

${ }_{410}$ In this section, the results of the inclined plane are presented. The input parameters and initial conditions of the flow are in table 1.

\begin{tabular}{|l|c|}
\hline Pile shape & Cylindrical \\
\hline Maximum pile height & $.061 \mathrm{~m}$ \\
\hline Major extent of the pile & $.0525 \mathrm{~m}$ \\
\hline Minor extent of the pile & $.0525 \mathrm{~m}$ \\
\hline Bed friction angle & $32.47^{\circ}$ \\
\hline Internal friction angle & $37.3^{\circ}$ \\
\hline Angle of incline & $38.5^{\circ}$ \\
\hline Physical time simulated & $90 \mathrm{sec}$ \\
\hline
\end{tabular}

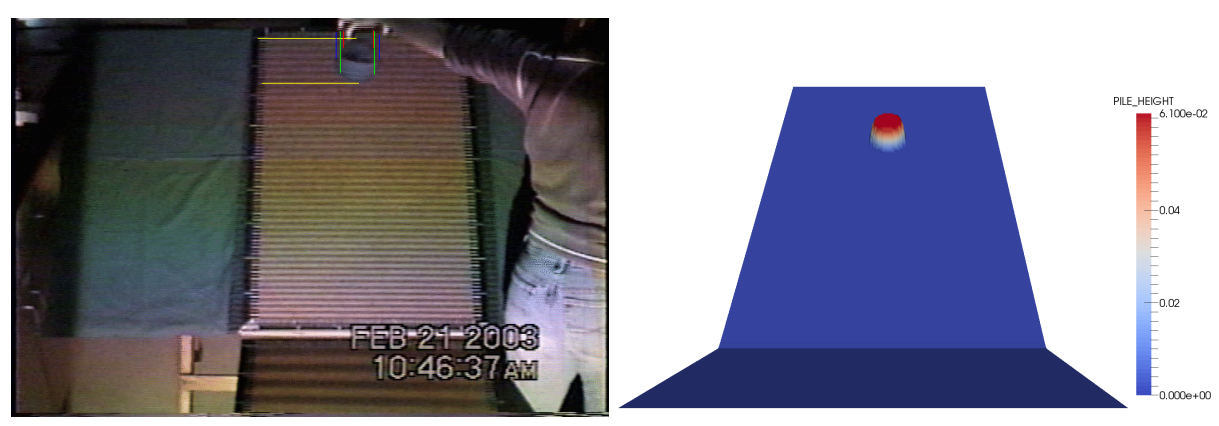
(a) Experimental setup
(b) Numerical simulation

Figure 7: Initial configuration of the pile on the incline. 

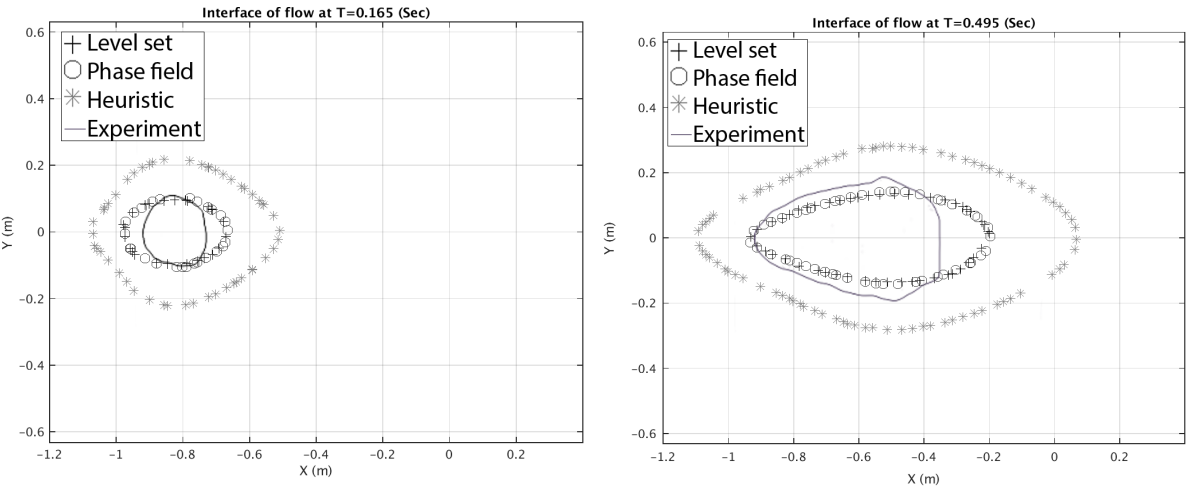

(a) $\mathrm{T}=0.165 \mathrm{sec}$

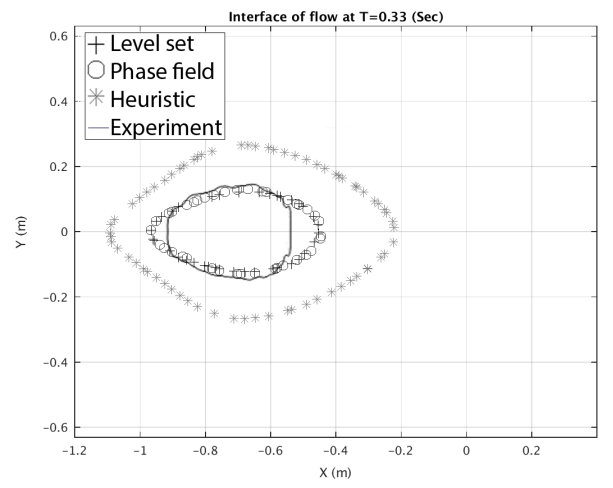

(b) $\mathrm{T}=0.33 \mathrm{sec}$

(c) $\mathrm{T}=0.495 \mathrm{sec}$

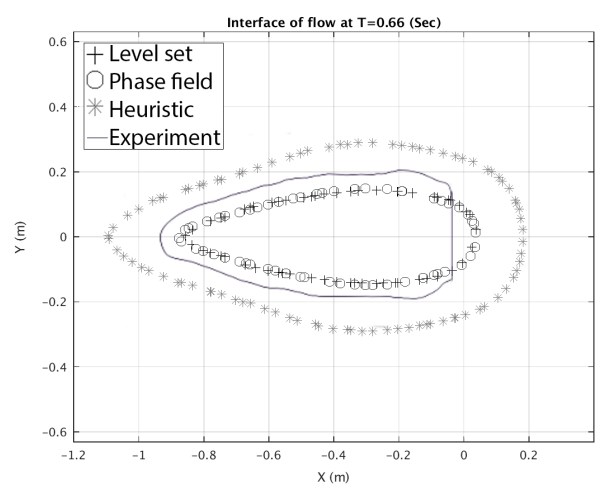

(d) $\mathrm{T}=0.66 \mathrm{sec}$

Figure 8: Pile height contour and interface location at different time steps.

The experimental results were reported in [55], and we have used them to verify numerical results in previous work. Figure 7 shows the initial configuration of the pile in both the experimental setup and the numerical study. As can be seen in the experimental setup, there are markers in the inclined plane that are used for measurements. Webb [55] used a digital camera and image processing techniques to compute the extents of the flow in $\mathrm{x}$ and $\mathrm{y}$ directions as well as its area and perimeter, and then compared the obtained results with the numerical simulations. The raw data from observation was re-analyzed in the course of this investigation and the actual flow depth data was updated to 
account for a small error in the original calculations reported in [55. We also plotted the interface of the flow for the three interface capturing schemes and the experimental results in figure 8 . The plotted interfaces in figure 8 are for ${ }_{425} \phi=0$ and $\Psi=0$ for the Phase Field and the Level Set methods respectively and $h=\sqrt[3]{V o l} \times$ GEOFLOW_TINY for the heuristic method where $V o l$ is the volume of flow and GEOFLOW_TINY $=.0001$, as was described in section 4.1.1.

Figure 8 shows that the results of the Eulerian interface capturing schemes are very similar for the inclined plane, and are closer to the experiment than the heuristic method. In all four plotted time steps, the heuristic method appears to move ahead of the true interface. It is possible to obtain better agreement by tuning the value of GEOFLOW_TINY but in that case we will end up with a GEOFLOW_TINY that depends on the particular flow rendering the use of TITAN for anything but hindcasts problematic. A primary goal of this study is to find a method that can predict the interface of a geophysical flow independent of the scale of the flow, and for this reason we selected two very different scales of flows (i.e flow over the inclined plane and Colima volcano), and the GEOFLOW_TINY that we use here have been tested in much of our previous work, and has showed reasonable results $[3,37$.

In figure 9a the extent of flow in $\mathrm{X}$ direction was plotted. This plot shows the length of flow during its travel. The maximum of the flow length happens when the flow reaches the horizontal plane. All of the methods are successful in predicting the time that flow reaches the horizontal plane, but like the interface results (figure 8) the heuristic method over-predicts the length of the flow. The

${ }_{445}$ Phase Field and the Level Set results are a better match with the experimental result.

The width of the flow or extent of the flow in $\mathrm{Y}$ direction can be seen in figure 9c. There is a much larger difference between the experiment and the Eulerian interface capturing methods results in this figure than the other results, and based on our previous work [37, we believe the main reason for this difference is due to the accuracy of the solver. In that work, we showed that with more accurate simulations using a higher order Discontinuous Galerkin method we can 
better capture the width of the flow. Future work will address the combination of such higher order methods and the interface capturing schemes.

The other measure we use to compare the results is the area of the flow. In figure $9 \mathrm{~b}$ one can see that the Eulerian methods capture the area of the flow fairly well, especially before the flow hits the horizontal surface while the heuristic method over-estimates the area of the flow. The last result is that of the perimeter of the flow (figure 9d), which shows a similar behavior to the other results that have been discussed earlier.

From these results, all three methods successfully overcome the thin layer problems (problems 1-4 section 1) for this simple problem, though the Eulerian interface capturing methods captured the flow with significantly higher accuracy than the heuristic method. 

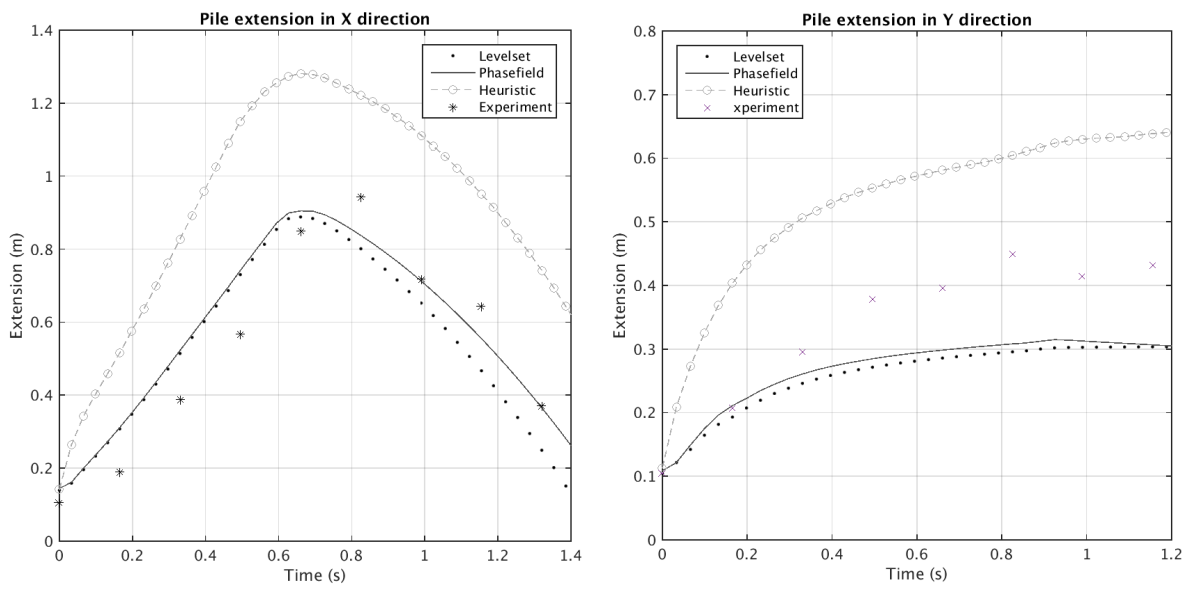

(a) Extension of pile in $\mathrm{X}$ direction.

(c) Extension of pile in $\mathrm{Y}$ direction.
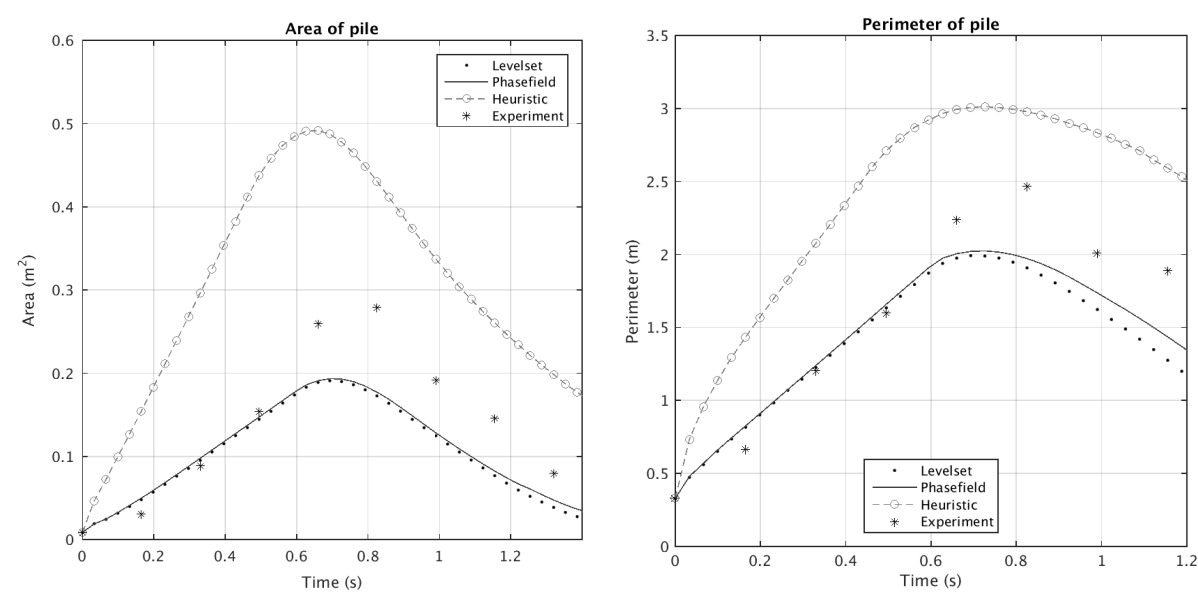

(b) Area of pile.

(d) Perimeter of pile.

Figure 9: Comparison of the methods for flow on inclined plane. 


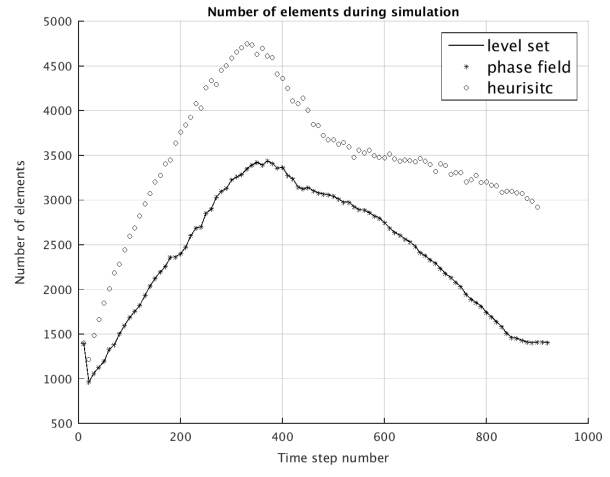

(a) Number of the elements for different method of interface capturing during the simulation.

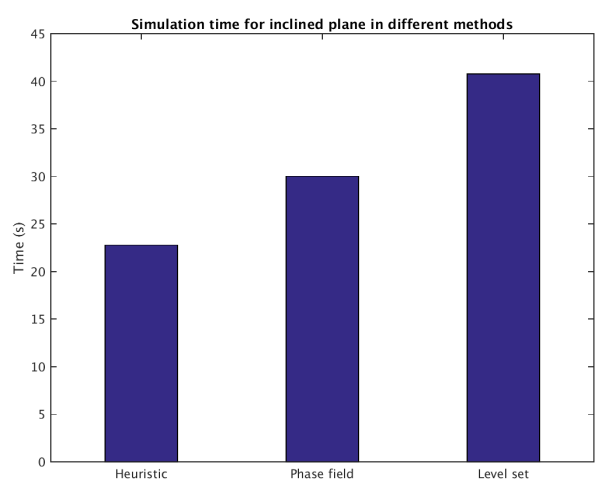

(b) Time spent for each simulation. 480

Figure 10: Comparison of the methods based on computational cost. figure 10. The first plot is the number of elements during the simulation and the second one is the wall clock time spent for each simulation. Since we are using the results of the interface capturing methods for adaptive mesh refinement as described in section 3.2 , the number of elements during the simulation changes and depends on the different interface capturing methods we use. Based on these results, as can be predicted from similarity of the results in the Eulerian interface capturing methods figures 9 and 8 , both Eulerian interface capturing methods have a very similar number of elements during the simulation while the interface capturing with the heuristic method results in a larger number 45 the total time needed to complete a simulation, figure $10 \mathrm{~b}$, is lowest for the heuristic method. Overall the heuristic method took $70 \%$ of the time required for the Phase Field method and only $55 \%$ of the time required for the Level Set method. All of the simulations were performed on one $\operatorname{Intel}(\mathrm{R}) \mathrm{Xeon}(\mathrm{R}) \mathrm{CPU}$ E3-1220 v3 3.10GHz. Clearly, there is a tradeoff in accuracy and cost. 


\subsection{Colima Volcano}

In this section, we compare our different interface capturing methods to field data from an eruption of the Colima volcano. The Colima volcano in Mexico is one of the most active volcanoes in North America, and this eruption happened on 16-17 April 1991 [56].

\begin{tabular}{|l|c|}
\hline Pile shape & Parabolic \\
\hline Location of pile in X & 644956.0 \\
\hline Location of pile in Y & 2157970.0 \\
\hline Maximum pile height & $30 \mathrm{~m}$ \\
\hline Major extent of the pile & $55 \mathrm{~m}$ \\
\hline Minor extent of the pile & $55 \mathrm{~m}$ \\
\hline Bed friction angle & $37^{\circ}$ \\
\hline Internal friction angle & $20^{\circ}$ \\
\hline Physical time simulated & $3600 \mathrm{sec}$ \\
\hline
\end{tabular}

The topography of the Colima volcano is such that small changes in the initial location of the pile leads to a completely different path of flow, so good performance of any of the interface capturing methods for this case promises a reliable method for other volcanoes. Unfortunately, only in very rare cases is data during a volcanic eruption available, and thus we cannot perform a direct comparison between the numerical results with time-resolved field data. Usually, for such applications, the deposit of the volcanic event is used to find the outline of the flow and to validate the numerical results.

In figure 12 we compared the results of the three methods with the outline of the flow from the eruption obtained from the field data [57]. In the numerical simulations, the outline of the flow is the boundary between wet and dry areas of the domain for whole the simulation. In other words, the points where the flow has passed are inside the outline, while the rest of the points are located outside of the outline of the flow.

As in the inclined plane case, we use the same definition for the interface. Thus $\phi=0$ and $\Psi=0$ are used for the Phase Field and the Level Set methods, 
respectively, and $h=\sqrt[3]{V o l} \times$ GEOFLOW_TINY is used for the heuristic method to determine the wet and dry areas.

The resolution of the digital elevation model (DEM) of Colima volcano that we use here is 5 meters, which is the finest DEM that we have ever used for this volcano. The outline of this eruption is available in [58. We used the Keyhole Markup Language (KML) to plot the results on real terrain information by using the Google earth application 12 .

In figure 11 one can see the plotted outline of the eruption on Colima volcano.

This outline is compared to the numerically determined results in figure 12 .

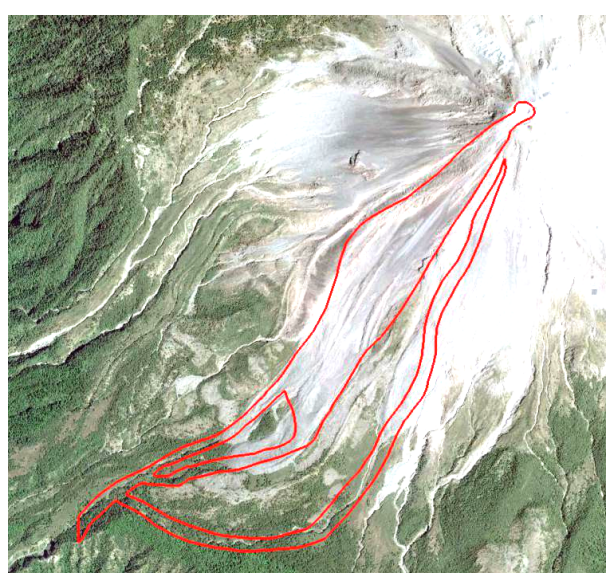

Figure 11: Outline of flow for Colima volcano for eruption 16-17 April 1991. 
(a) Outline from Heuristic Method.

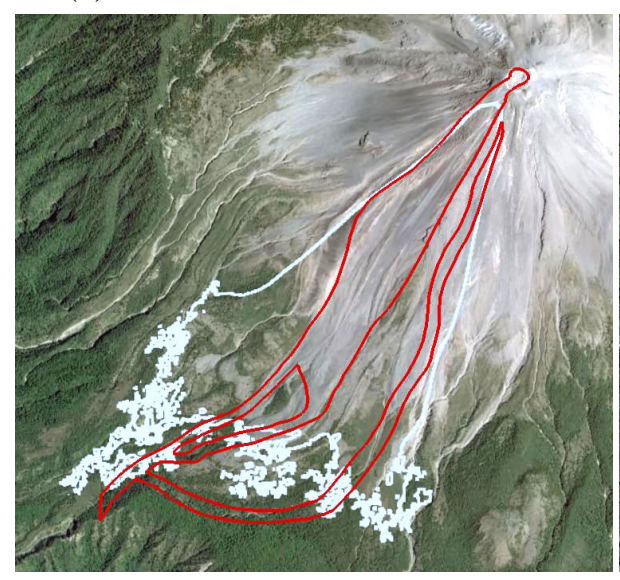

(b) Outline from Level Set Method.

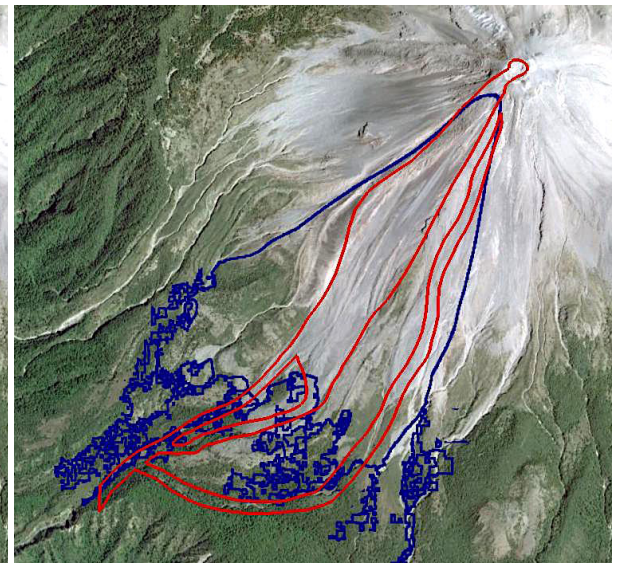

(c) Outline from Phase field Method.

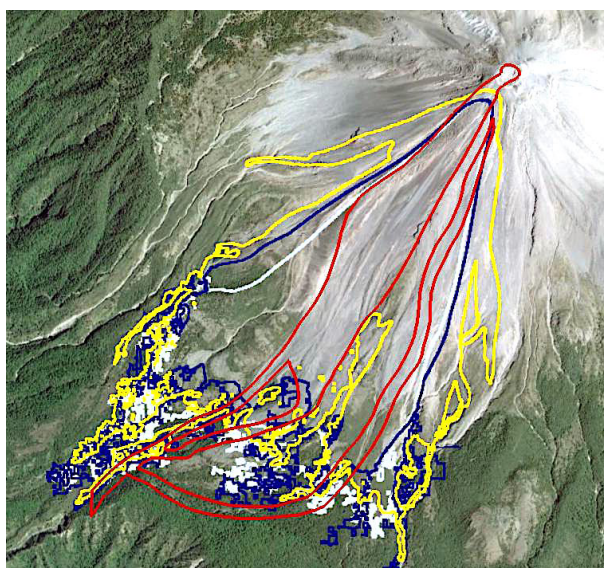

(d) Comparison of all three methods.

Figure 12: Comparison of the obtained outline of flow from Heuristic, Level Set, and Phase field methods for Colima Volcano.

Results of figure 12 show that all of the methods are successful in controlling the thin layer problem. The length of the flow has been captured in all of the methods very well. This is very important for generating hazard maps. The 515 width of the flow is also reasonable in all of the three methods, although the Eulerian methods are slightly more accurate. The heuristic method captures the separation of the flow into two channels that can be seen in the field deposit 
data. The heuristic method also indicates the presence of two small channels that are not present in the field outline. Note that we are comparing deposits to field data.
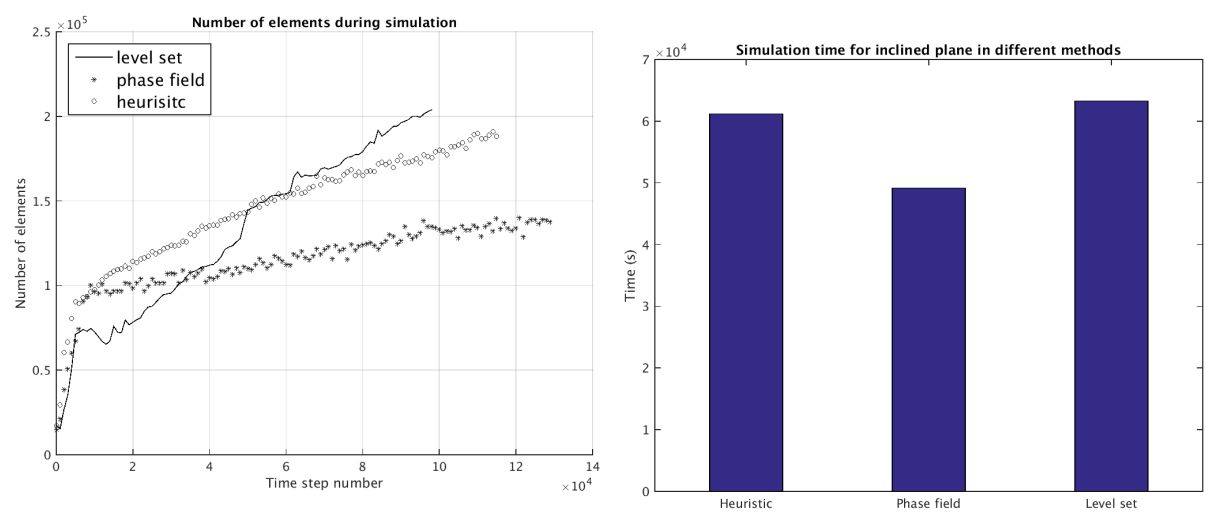

(a) Number of the elements for different method of interface capturing during the (b) Time spent for each simulation. simulation.

Figure 13: Comparison of the methods based on computational cost.

The computational cost comparison of the methods for the Colima volcano simulations is shown in figure 13 We used $16 \times 2.27 \mathrm{GHz} \operatorname{Intel}(\mathrm{R}) \mathrm{Xeon}(\mathrm{R})$ L5520 for this case. Figure $13 \mathrm{a}$ shows the number of the elements during the simulation for the three methods. This figures shows the heuristic method and level set have a higher number of elements than the phase field method, and both require a higher number of iterations to perform $1 \mathrm{hr}$ physical time simulation. The right figure (figure 13b shows the required time for the simulation. In this figure, we can see all of the methods take almost the same amount of computational time, which means that although the Level Set and the Phase ${ }_{530}$ Field methods are more computationally expensive per time step, the lower number of elements and lower number of iterations help them to have the same computational cost as the heuristic method. 


\section{Conclusions}

The numerical solution of the Savage-Hutter (and similar "shallow-water") equations have historically been plagued by several interrelated numerical difficulties which are collectively characterized by a non-physically thin-layer extending large distances from the realistic main body of the flow. In the best case, this "thin-layer problem" means a "no flow" boundary line must be arbitrarily drawn at some given depth contour. In the worst case, it can cause 540 severe numerical instability that prevents any simulation of a particular event. In this paper, we have described some features of the thin-layer problem, some underlying causes that are common to virtually all numerical solution methodologies for SW equations. We presented a heuristic method and compared it to two Eulerian interface capturing approaches, namely phase field and level set based methods, that mitigate this problem. In the heuristic method we used a threshold to distinguish between the wet and dry areas, and in the level set and phase field methods, we solved a transport equation that implicitly represents the interface of the flow. Then we used the result of a previous step for mesh refinement in all of the three solvers to control the thin layer problem and other related problems. Moreover, in the heuristic approach, we also used this result for interface reconstruction and the adjustment of the flux. We implemented these thin-layer control strategies in TITAN2D, our high performance finite volume solver of the depth-averaged granular flow equations. Numerical simulations were performed for two different geophysical mass flows. To test the solvers, numerical experiments were conducted for an inclined plate and the Colima volcano. For the first case we compared the numerical results with experimental results, and for the second case we compared the results with field data. These analyses show that with all of the approaches, we not only prevented the loss of numerical stability but also demonstrated behavior that is consistent with observations of the flows. In the case of the inclined plane the phase field and level sets also appear to capture flow outlines more accurately. On the basis of these very positive results, we conclude that our thin-layer con- 
trol strategy, and interface capturing approach provides sufficient benefit. While all of these approaches to thin-layer mitigation were developed in the context of TITAN2D and granular flows, much of it should be appropriate for use in depth-averaged flow solvers with different numerical schemes and physics.

\section{Acknowledgements}

We acknowledge the Colima deposit and experiment data made available to us by Prof. M. I. Bursik and for helpful discussions on the use of that data. The work described above was funded by NSF awards ACI 1339765 and IDR 1131074.

\section{References}

口 [1] S. B. Savage, K. Hutter, The motion of a finite mass of granular material down a rough incline, Journal of Fluid Mechanics 199 (-1) (1989) 177. doi:10.1017/S0022112089000340.

URL http://journals . cambridge.org/article_S0022112089000340

[2] M. E. Eglit, Y. I. Sveshnikova, Matematicheskoye modelirovaniye snezhnykh lavin; mathematical modeling of snowavalanches, Materialy Glyatsiologicheskikh Issledovaniy, Khronika Obsuzhdeniya 38 (1980) 79-84.

[3] A. K. Patra, a. C. Bauer, C. C. Nichita, E. B. Pitman, M. F. Sheridan, M. Bursik, B. Rupp, a. Webber, a. J. Stinton, L. M. Namikawa, C. S.

口 Renschler, Parallel adaptive numerical simulation of dry avalanches over natural terrain, Journal of Volcanology and Geothermal Research 139 (1-2) (2005) 1-21. doi:10.1016/j.jvolgeores.2004.06.014. S0377027304002288

[4] E. F. Toro, Shock-Capturing Methods for Free-Surface Shallow Flows, Wiley, New York, 2001. 
[5] K. R. Dalbey, Predictive Simulation and Model Based Hazard Maps, Ph.D. thesis, University at Buffalo (2009).

[ [6] S. C. Medeiros, S. C. Hagen, Review of wetting and drying algorithms for numerical tidal flow models, International Journal for Numerical Methods in Fluids 71 (4) (2013) 473-487. doi:10.1002/fld.3668.

URL http://doi.wiley.com/10.1002/fld.3668

595 [7] A. Balzano, Evaluation of methods for numerical simulation of wetting and drying in shallow water flow models, Coastal Engineering 34 (1998) $83-107$.

1] URL http://www.sciencedirect.com/science/article/pii/ S0378383998000155

${ }_{600}^{0}$ [8] F. Aureli, a. Maranzoni, P. Mignosa, C. Ziveri, A weighted surface-depth 1 gradient method for the numerical integration of the 2D shallow water equations with topography, Advances in Water Resources 31 (7) (2008) 962-974. doi:10.1016/j.advwatres.2008.03.005.

1) URL http://linkinghub.elsevier.com/retrieve/pii/

605 S0309170808000468

n [9] S. Bunya, E. J. Kubatko, J. J. Westerink, C. Dawson, A wetting and drying

n. treatment for the RungeKutta discontinuous Galerkin solution to the shallow water equations, Computer Methods in Applied Mechanics and Engineering 198 (17-20) (2009) 1548-1562. doi:10.1016/j.cma.2009.01.008 URL http://linkinghub.elsevier.com/retrieve/pii/ S0045782509000383

[10] V. Casulli, A high-resolution wetting and drying algorithm for free-surface hydrodynamics, International Journal for Numerical Methods in Fluids 60 (August 2008) (2009) 391-408.

615 [11] G. Kesserwani, Q. Liang, Locally Limited and Fully Conserved RKDG2 Shallow Water Solutions with Wetting and Drying, Journal of Scientific 

the Venice lagoon, Computers \& Geosciences 33 (4) (2007) 476-496. doi:10.1016/j.cageo.2006.07.009.

[13] M. Castro, A. Ferreiro Ferreiro, J. García-Rodríguez, J. González-Vida, q J. Macías, C. Parés, M. Elena Vázquez-Cendón, The numerical treatment 1. of wet/dry fronts in shallow flows: application to one-layer and two-layer systems, Mathematical and Computer Modelling 42 (3-4) (2005) 419-439. doi:10.1016/j.mcm.2004.01.016 URL http://dx.doi.org/10.1016/j.mcm.2004.01.016

[14] F. H. Harlow, J. E. Welch, Numerical Calculation of Time-Dependent Viscous Incompressible Flow of Fluid with Free Surface, Physics of Fluids 8 (1965) 2182-2189.

635 [15] L. Cheng, S. Armfield, A simplified marker and cell method for unsteady flows on non-staggered grids, International Journal for Numerical Methods in Fluids 21 (1) (1995) 15-34. doi:10.1002/fld.1650210103 URL http://dx.doi.org/10.1002/fld.1650210103

[16] L. C. Wrobel, C. A. Brebbia, Computational Modelling of Free and Moving 640 Boundary Problems: Fluid flow, Vol. 1, Walter de Gruyter, 1991.

[17] J. Glimm, J. W. Grove, X. L. Li, K.-M. Shyue, Y. Zeng, Q. Zhang, Three Dimensional Front Tracking, SIAM J. Sci. Comp 19 (1995) 703-727.

[18] S. O. Unverdi, G. Tryggvason, A Front-tracking Method for Viscous, Incompressible, Multi-fluid Flows, J. Comput. Phys. 100 (1) (1992) 25-37. 
645

[19] S. Osher, J. A. Sethian, Fronts propagating with curvature-

n. dependent speed: Algorithms based on Hamilton-Jacobi formulations, Journal of Computational Physics 79 (1) (1988) 12-49.

650

$[20]$ ods in Fluid Mechanics, Annual Review of Fluid Mechanics 30 (1) (1998)

655

[24] V. R. Gopala, B. G. M. van Wachem, Volume of fluid methods for immiscible-fluid and free-surface flows, Chemical Engineering Journal.

[25] J. W. Cahn, J. E. Hilliard, Free Energy of a Nonuniform System. I. Interfacial Free Energy, The Journal of Chemical Physics 28 (2).

670

[21] C. W. HIRT, B. D. NICHOLS, Volume of fluid/VOF/ method for the dynamics of free boundaries, Journal of Computational Physics 39 (1) (1981) $201-225$.

$[22]$ D. L. Youngs, Time-dependent multi-material flow with large fluid distortion, in: K. W. Morton, M. J. Baines (Eds.), Numerical Methods for Fluid Dynamics, Academic Press, 1982, pp. 273-285.

[23] D. Gerlach, G. Tomar, G. Biswas, F. Durst, Comparison of volume-offluid methods for surface tension-dominant two-phase flows, International Journal of Heat and Mass Transfer 49 (3-4) (2006) 740-754.

[26] J. W. Cahn, Free Energy of a Nonuniform System. II. Thermodynamic Basis, The Journal of Chemical Physics 30 (5). 
[27] X. Yang, J. J. Feng, C. Liu, J. Shen, Numerical simulations of jet

n pinching-off and drop formation using an energetic variational phase-field method, Journal of Computational Physics 218 (1) (2006) 417-428.

65 doi:http://dx.doi.org/10.1016/j.jcp.2006.02.021.

口 URL http://www.sciencedirect.com/science/article/pii/ S002199910600091X

[28] M. Quecedo, M. Pastor, A reappraisal of Taylor-Galerkin algorithm for drying-wetting areas in shallow water computations, International Journal for Numerical Methods in Fluids 38 (6) (2002) 515-531.

[29] J. Kim, S. Lee, Y. Choi, A conservative Allen Cahn equation with a space time dependent Lagrange multiplier, International Journal of Engineering Science 84 (2014) 11-17. doi:10.1016/j.ijengsci.2014.06.004.

[30] K.Hutter, M. Siegel, S. B. Savage, Y. Nohguchi, Two-dimensional spreading of a granular avalanche down an inclined plane part I. Theory, Acta Mechanica 100 (1993) 37-68.

[31] R. M. Iverson, The physics of debris flows, Reviews of Geophysics 35 (1997) 245-296. doi:10.1029/97RG00426.

[32] J. M. N. T. Gray, M. Wieland, K. Hutter, Gravity-driven free surface flow of granular avalanches over complex basal topography, Proceedings of the Royal Society A: Mathematical, Physical and Engineering Sciences 455 (1985) (1999) 1841-1874. doi:10.1098/rspa.1999.0383.

1 URL http://rspa.royalsocietypublishing.org/cgi/doi/10.1098/ rspa.1999.0383

${ }_{695}[33]$ R. P. Denlinger, R. M. Iverson, Flow of variably fluidized granular masses a across three-dimensional terrain: 2. Numerical predictions and experimen-

1. tal tests, Journal of Geophysical Research 106 (B1) (2001) 553. doi: 10.1029/2000JB900330.

URL http://doi .wiley.com/10.1029/2000JB900330 
[34] S. P. Pudasaini, K. Hutter, Rapid shear flows of dry granular masses down curved and twisted channels, Journal of Fluid Mechanics 495 (2003) 193208.

[35] S. B. Savage, R. M. Iverson, Surge dynamics coupled to pore-pressure evolution in debris flows, Debris-Flow Hazards Mitigation: Mechanics, Predic-

[37] A. K. Patra, C. Nichita, A. Bauer, E. Pitman, M. Bursik, M. Sheridan,

घ Parallel adaptive discontinuous Galerkin approximation for thin layer avalanche modeling, Computers \& Geosciences 32 (7) (2006) 912-926. doi:10.1016/j.cageo.2005.10.023 URL http://linkinghub.elsevier.com/retrieve/pii/ S0098300405002736

[38] E. F. Toro, Riemann Solvers and Numerical Methods for Fluid Dynamics: A Practical Introduction, Springer, 2009. URL http://books .google.com/books?id=SqEjXOum8o0C

[39] L. Fraccarollo, E. F. Toro, Experimental and numerical assessment of the shallow water model for two dimensional dam-break type problems, Journal of Hydraulic Research 33 (1995) 843-864.

[40] Y. Tai, S. Noelle, J. Gray, K. Hutter, Shock-Capturing and Front-Tracking Methods for Granular Avalanches, Journal of Computational Physics 175 (1) (2002) 269-301. doi:10.1006/jcph.2001.6946

URL http://linkinghub.elsevier.com/retrieve/pii/ S0021999101969463 
[41] L. Q. Chen, Phase Field Models for Microstructure Evolution, Annual Review of Materials Research 32 (1) (2002) 113-140.

[42] W. J. Boettinger, J. a. Warren, C. Beckermann, A. Karma, Phase -Field Simulation of Solidification, Annual Review of Materials Research 32 (1) (2002) 163-194. doi:10.1146/annurev.matsci.32.101901.155803.

q URL http://www.annualreviews.org/doi/abs/10.1146/annurev. matsci.32.101901.155803

[43] J. Kim, Phase-Field Models for Multi-Component Fluid Flows, Com-

๓ mun. Comput. Phys 12 (3) (2012) 613-661. doi:10.4208/cicp.301110. $040811 \mathrm{a}$

URL http://158.182.10.53/openaccess/v12_613.pdf

[44] L. Bronsard, R. V. Kohn, On the slowness of phase boundary motion in one space dimension, Communications on Pure and Applied Mathematics 43 (8) (1990) 983-997. doi:10.1002/cpa.3160430804.

URL http://dx.doi.org/10.1002/cpa.3160430804

[45] R. G. Larson, The Structure and Rheology of Complex Fluids Topics in Chemical Engineering, OUP USA, 1999.

URL http://books.google.de/books?id=Vt9fw_pf1LUC

[46] S. Balay, S. Abhyankar, M. F. Adams, J. Brown, P. Brune, K. Buschelman, V. Eijkhout, W. D. Gropp, D. Kaushik, M. G. Knepley, L. C. McInnes, K. Rupp, B. F. Smith, H. Zhang, PETSc Users Manual, Tech. Rep. ANL95/11 - Revision 3.5, Argonne National Laboratory (2014).

URL http://www.mcs .anl.gov/petsc

[47] C. E. Kees, I. Akkerman, M. W. Farthing, Y. Bazilevs, A conservative level 755 set method suitable for variable-order approximations and unstructured 
meshes, Journal of Computational Physics 230 (12) (2011) 4536-4558. doi: $10.1016 / j \cdot j c p .2011 .02 .030$

URL http://dx.doi.org/10.1016/j.jcp.2011.02.030

[48] F. Losasso, R. Fedkiw, S. Osher, Spatially adaptive techniques for level

प

[49] J. a. Sethian, P. Smereka, L Evel S Et M Ethods for F Luid I Nter-

[50] C. Li, R. Huang, Z. Ding, J. C. Gatenby, D. N. Metaxas, J. C. Gore,

[52] D. L. Chopp, Some Improvements of the Fast Marching Method, SIAM

[53] M. Sussman, P. Smereka, S. Osher, A Level Set Approach for Computing

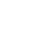
S004579300500174X faces, Annual Review of Fluid Mechanics 35 (1) (2003) 341-372. doi:10.1146/annurev.fluid.35.101101.161105

\section{URL http://www.annualreviews.org/doi/abs/10.1146/annurev.} fluid.35.101101.161105 A level set method for image segmentation in the presence of intensity inhomogeneities with application to MRI, IEEE Transactions on Image Processing 20 (7) (2011) 2007-2016. doi:10.1109/TIP.2011.2146190.

[51] J. Lie, M. Lysaker, X.-C. Tai, A variant of the level set method and applications to image segmentation, Mathematics of Computation 75 (255) (2006) 1155-1175. doi:10.1090/S0025-5718-06-01835-7. Journal on Scientific Computing 23 (1) (2001) 230-244. doi:10.1137/ S106482750037617X Solutions to Incompressible Two-Phase Flow, Journal of Computational Physics 114 (1) (1994) 146-159. doi:10.1006/jcph.1994.1155.

URL http://www.sciencedirect.com/science/article/pii/ http://www.sciencedirect.com/science/article/pii/ S0021999184711557 
[54] D. Adalsteinsson, J. Sethian, The Fast Construction of Extension Velocities in Level Set Methods, Journal of Computational Physics 148 (1) (1999) 2-22. doi:10.1006/jcph.1998.6090

URL http://linkinghub.elsevier.com/retrieve/pii/ S0021999198960909

[55] A. Webb, Granular flow experiments to validate numerical flow model, Titan2D, Ph.D. thesis, University at Buffalo (2004).

[56] S. J. Charbonnier, R. Gertisser, Field observations and surface

1 characteristics of pristine block-and-ash flow deposits from the 2006 eruption of Merapi Volcano, Java, Indonesia, Journal of Volcanology and Geothermal Research 177 (4) (2008) 971-982. doi:http://dx.doi.org/10.1016/j.jvolgeores.2008.07.008. URL http://www.sciencedirect.com/science/article/pii/ S0377027308004241

[57] B. Rupp, M. Bursik, L. Namikawa, A. Webb, A. K. Patra, R. Saucedo, J. L. Macías, C. Renschler, Computational modeling of the 1991 block and ash fows at Colima Volcano, Mexico, Geological Society of America Special Paper 4022402 (11) (2006) 223-237. doi:10.1130/2006.2402(11).

[58] L. M. Namikawa, Multiple Representations of Elevation for Dynamic Process Modeling, Ph.D. thesis, Department of Geography, University at Buffalo (Jul. 2006). 